IDEAs in Ectlogy ANd Evalutian 12: 28-34, 2019

doi: $10.24908 /$ iee.2019.12.5.c

(C) 2019 The Author

Received 12 July 2019; Accepted 16 September 2019

Commentary

\title{
Discoveries and novel insights in ecology using structural equation modeling
}

\author{
Daniel C. Laughlin and James B. Grace
}

Daniel C. Laughlin (daniel.laughlin@uwyo.edu), Department of Botany, University of Wyoming, Laramie, WY, USA

James B. Grace, U.S. Geological Survey, Lafayette, LA, 70506 USA

\begin{abstract}
As we enter the era of data science (Lortie 2018), quantitative analysis methodologies are proliferating rapidly, leaving ecologists with the task of choosing among many alternatives. The use of structural equation modeling (SEM) by ecologists has increased in recent years, prompting us to ask users questions about their experience with the methodology. Responses indicate an enthusiastic endorsement of SEM. Two major elements of respondent's experiences seem to contribute to their positive response, (1) a sense that they are obtaining more accurate explanatory understanding through the use of SEM and (2) excitement generated by the discovery of novel insights into their systems. We elaborate here on the detection of indirect effects, offsetting effects, and suppressed effects, and demonstrate how discovering these effects can advance ecology.
\end{abstract}

\section{The proliferation of computational tools}

Science is entering an era where aspirations for what can be learned from data are increasing. Discussions of emergent methodological trends in computational biology highlight many benefits that can come from the integration of new data sources and analytical tools in the scientific process (Lortie 2018). While there is much excitement about the potential for novel insights that can come from new data types and new forms of analysis, quantitative analysis methodologies are proliferating rapidly, leaving ecologists with the task of choosing among many alternatives.

We feel that it is vitally important for scientists who use or rely on analytical methods to keep in mind the fundamental distinction between explanatory and descriptive methodologies. While many still routinely attempt to draw scientific cause-effect inferences using statistical tools not designed for that purpose, ecologists are increasingly gaining familiarity with explanatory modeling methods. Randomized experiments remain the most basic approach to testing explanations. It is of course recognized that many ecological phenomena of interest involve spatial and temporal scales well beyond the feasibility or ethics of experimental manipulation. It is less widely recognized that classical methods such as ANOVA are strictly reductionist, providing inferences only for the variables that were manipulated but not for other factors in the system that convey the effects of the manipulations to the observed responses.

Another, more flexible methodology is structural equation modeling (SEM) (Shipley 2002, Grace 2006). SEM can be profitably used with data from randomized experiments (Grace et al. 2009), though it is often used to evaluate explanatory hypotheses against observational data. While certainly not a new means of analysis, SEM continuously evolves and incorporates the latest relevant statistical developments (Grace et al. 2012). The use of structural equation modeling has increased over time as scientists have increasingly strived to develop an integrative understanding of ecological systems (Figure $1)$.

As ecologists are presented with a proliferation of new analytical tools, they often only learn about them through their readings and conversations with colleagues. Because we feel that SEM is particularly valuable for its ability to encapsulate scientist's ideas as well as to reveal novel and unanticipated findings, we thought it would be valuable to conduct a survey of ecologists who have used 


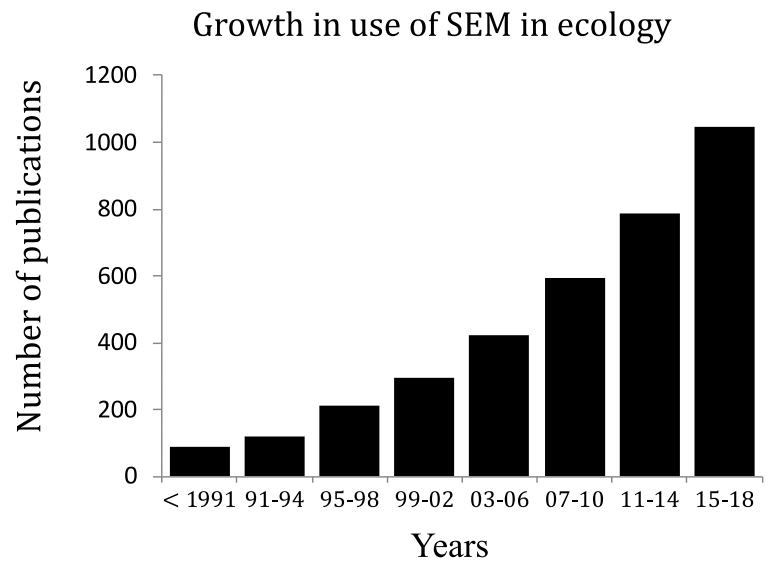

Figure 1. Change over time in the number of publications in journals whose names include "ecology" or "ecological" and whose texts include either "path analysis" or "structural equation", based on Google Scholar.

SEM in their papers, report their experiences, and illustrate some of the types of findings they found most surprising.

\section{Some Distinctive Features of SEM}

The ability of a methodology to have distinctive value will be linked to its distinctive features. As an illustration of this principle, there are many types of regression models, but they all have the same general weaknesses with regard to explanatory interpretation. Regression models are based on the fundamental equational form,

$$
\mathbf{Y}=f(\mathbf{X}), \quad \text { Equation } 1
$$

where $\mathbf{Y}$ is a vector of response variables (or single response variable), $\mathbf{X}$ is a vector of predictors, and $f$ is one of many functional forms, ranging from General Linear Models to Generalized Linear Mixed Models (Fox 2015). Any attempt to interpret the effects of individual $x$ variables in this model is influenced (and typically negated) by uninterpreted correlations among them. As a general principle, it is only possible to interpret the effects of individual predictors in a correlated set if there is an explicit theory about why they are correlated (randomization solves this problem if it successfully isolates the $x$ of causal interest from all other $x \mathrm{~s}$ ).

Structural equation models (SE models) are based on the fundamental equational form,

$$
\mathbf{Y}=f(\mathbf{X}, \mathbf{Y}) . \quad \text { Equation } 2
$$

In this case, $\mathbf{Y}$ is a vector of response variables in a network of relationships, $\mathbf{X}$ is a vector of exogenous variables, and $f$ is a set of functional forms. SE models are designed to allow for the representation of scientific hypotheses in the form of causal probabilistic networks (Grace et al. 2014). It is this network that permits and requires linkage to theory and hypotheses to represent explicitly causal structures.

By permitting the specification of an indirect effect in a causal chain involving three variables, $A, B$, and $C$, e.g., $A \rightarrow B \rightarrow C$, it is possible to represent an explicit mechanism within the model (Figure 2, top panel). What is being hypothesized is that $B$ is the mechanistic piece that conveys the effects of $A$ on $C$. We may think we have an explanation for the direct links in a model (all effects in a regression model are direct), but the explanation is not explicit nor is it testable. Tests of conditional independence allow us to discover that the specified causal chain is either unsupported or incomplete. When unsupported, we may conclude that there is no empirical support for the proposed mediation mechanism. When incomplete, we conclude that there is some additional mechanism whereby $A$ influences $C$ that is not through $B$. This latter finding is where we discover a new mechanism operating that was unsuspected (a mechanism whereby $A$ influences $C$ independent of $B$ ), and which is only revealed when the network contains mediation hypotheses. Many other surprises can also be revealed through the use of SEM, a few of which we discuss below.

To give a sense of how these elements can be incorporated into a real-world application, Figure 3 shows a SE model of the effects of human activities on the biotic integrity of wetlands that was evaluated for Acadia National Park in Maine, USA (Grace et al. 2012). There are some elements of this model beyond the basics described in the preceding paragraphs. SEM is designed to allow investigators to incorporate their preexisting knowledge into a testable hypothesis. It also allows for the incorporation of latent/hypothesized variables (shown in ovals) in addition to observed variables (in boxes). This feature permits us to consider whether the influences of human activities on multiple measures of biotic integrity can be interpreted as generalized responses to the mediating processes of nutrient loading and altered hydrology. While we will not elaborate on the details of this study, one of the most highly appreciated aspects of SEM is the use of diagrams to simultaneously represent both the scientific ideas and the network of equations used to test those ideas. By performing the tests of conditional independence mentioned above, the investigators discovered that there appear to be additional processes whereby human activities degrade integrity beyond those specified in the initial model. 


\section{Indirect effect}

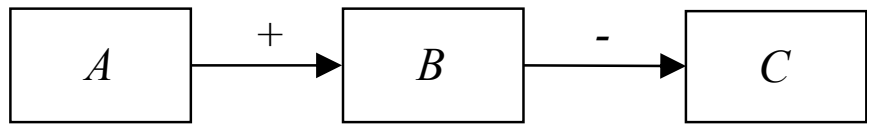

Offsetting effect

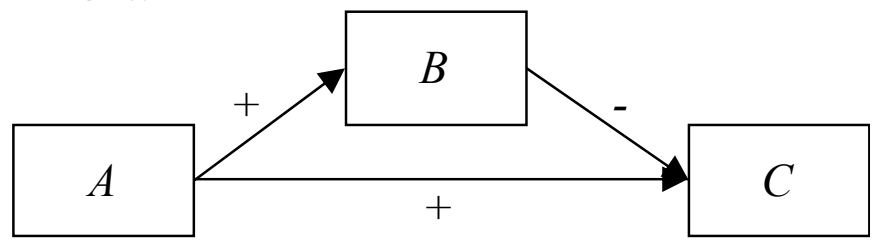

Figure 2. Three types of effects that can lead to discovery or novel insights in ecology, illustrated using three quantities, $A, B$, and $C$. The signs in the Indirect effect example (top panel) are not important but are consistent with the simulated example in the $\mathrm{R}$ Markdown file found at in the github repository https://github.com/danielLaugh lin/semDiscoveries. The signs in the Offsetting (middle panel) and Suppressed effect (bottom panel) examples are critical to their operation and relate directly to the examples in the text and the R Markdown file.

\section{Suppressed effect}

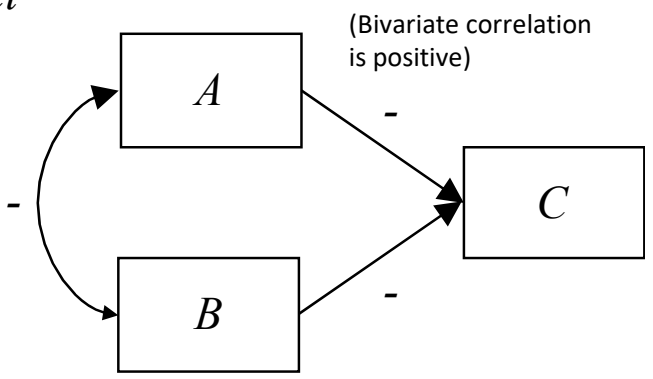

\section{A survey of SEM users}

We surveyed users of SEM that have published their work in ecological journals to gauge whether SEM was instrumental in improving their understanding of their ecological system. We asked questions about the individual's history of use with SEM, whether they thought the applications were successful, what they found useful, if they found results they could not have found using classical techniques, and what they think of the methodology generally.

We received 110 responses to the online survey. Survey results reported that $75 \%$ of the respondents had used SEM more than once. Eighty percent of respondents agreed with the statement that 'the methodology is useful for ecological studies under a broad range of conditions and should be used more commonly than it currently is.' Importantly, when asked 'Has SEM provided you with discoveries or novel insights into your system that you would not have discovered if you took a univariate approach to data analysis?', an overwhelming 93\% of respondents answered 'yes' to this question. Seventy percent of respondents elaborated on their answer and provided details about what they specifically learned.

Three common themes emerged from the personalized responses about ways that SEM has led them to discoveries or novel insights about their system. These themes included 1) identification of indirect relationships that were mediated through another variable, 2) the elucidation of offsetting relationships, and 3) the discovery of suppressed relationships. Ecologists are very familiar with the concept of an indirect relationship because they form a core component of many ecological theories. However, offsetting and suppressed effects are much less well understood and often viewed suspiciously. In this paper, we elaborate further on the test of mediation, then we describe the nature of offsetting and suppressed effects and provide examples from the recent ecological literature to demonstrate their importance. We also provide $\mathrm{R}$ code that simulates these effects to make these ideas more tractable. The code is available as an $\mathrm{R}$ Markdown file at the following github 
repository: https://github.com/danielLaughlin/semDis coveries.

\section{Three types of discoveries and novel insights}

\section{Indirect effects}

As described above, one of the most valuable attributes of SEM is its capacity to partition effects into direct and indirect pathways and the ability to explicitly test for mediation. We can describe various possibilities using only three variables. The simplest example of an indirect effect is when a predictor variable $A$ has an indirect effect on response variable $C$, mediated through its effect on response variable $B$ (Figure 2, top panel). The requirement for the test of mediation is that the product of the two path coefficients that link $A$ to $C$ through $B$ must be statistically indistinguishable from the observed bivariate (marginal) correlation coefficient between $A$ and $C$. See the accompanying R Markdown file for a simulated example.

Indirect effects are not only ubiquitous in ecological theories, but the test of mediation allows for explicit tests of mechanism. Let us turn to an example from the literature. Godschalx et al. (2015) studied the relationships between rhizobia, extrafloral nectaries, and ant abundance on lima bean. Lima bean associates with nitrogen fixing rhizobia to enhance resource uptake and also associates with ants to enhance defense. The researchers discovered that rhizobia have a negative indirect effect on plant defense by reducing the abundance of extrafloral nectaries that provide ants with a reward for defending the plant. Rhizobia decrease nectary abundance, but nectaries enhance ant abundance, so rhizobia indirectly reduce plant defenses by reducing nectary production.

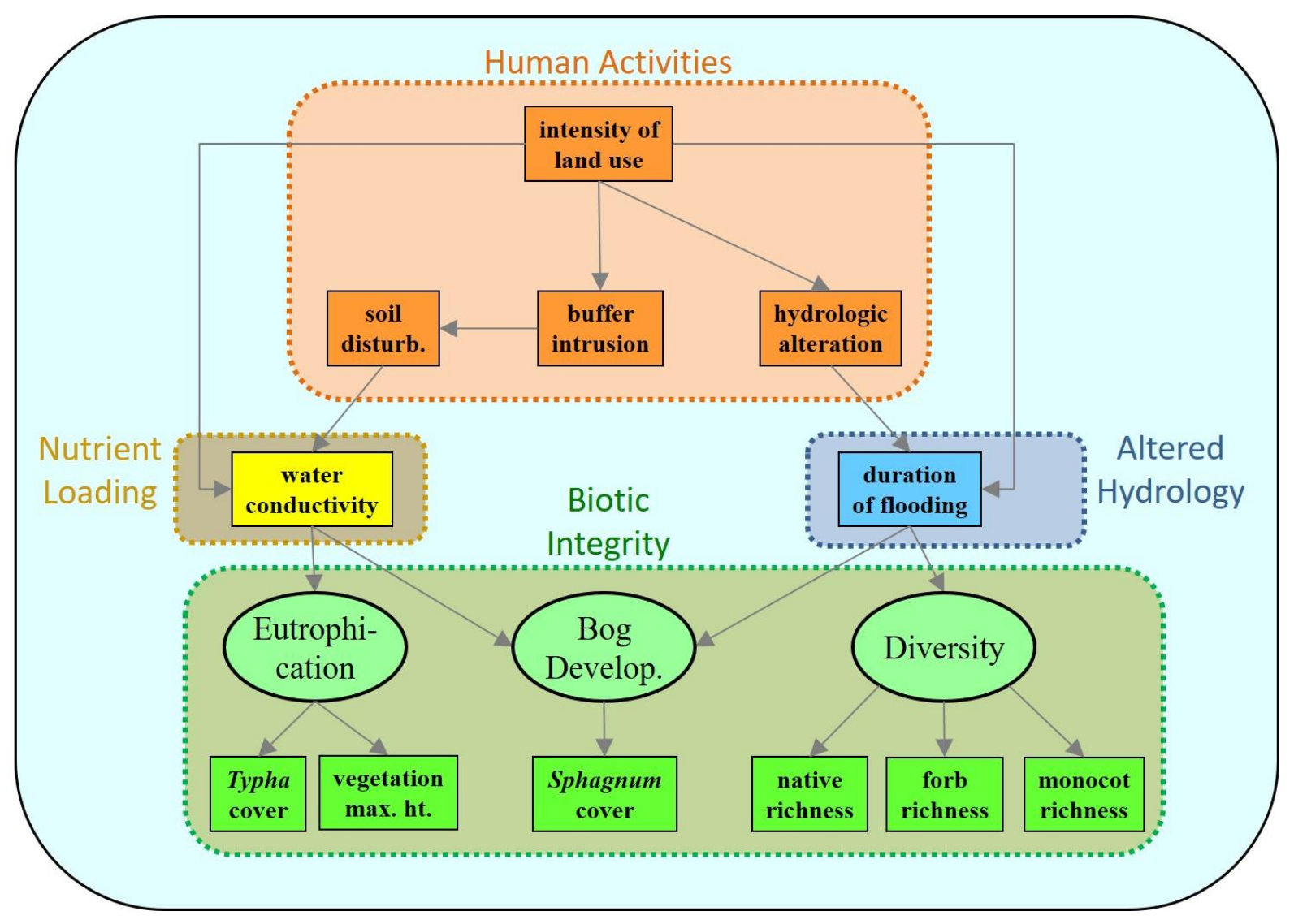

Figure 3. Example structural equation model representing a complex hypothesis about the effects of human activities around wetlands at Acadia National Park on components of biotic integrity. Boxes with solid lines represent observed measured variables. Ovals with solid lines represent generalized latent variables. Dashed line shapes show the relationships between variables and the broad concepts to which they belong. The general hypothesis being represented is that the influences of Human Activities on Biotic Integrity occurs through increased nutrient loads and a prolonged duration of flooding. 
In many cases, it is failures of the test of mediation that produce evidence for additional mechanisms representing novel findings. Gough and Grace (1999) tested the widely supported idea that fertilization decreases plant diversity through increased biomass and increased light competition. Results showed support for that causal chain, but also showed a very large negative effect on diversity independent of increases in plant biomass and shading. Several later SEM studies showed the same type of result and further provided evidence of a dramatic negative effect of soil acidification on diversity associated with nutrient additions to the system (Clark et al. 2007, Chen et al. 2013). Such novel insights into these complex interactions are not possible without an explicit test of mediation.

\section{Offsetting effects}

Offsetting effects are one of the least well understood and most mistrusted results in structural equation modeling, yet they are both common and insightful. Offsetting effects occur when two variables are linked by two different pathways that have net opposing signs. For example, $A$ may have a direct positive effect on $C$, but the indirect effect of $A$ on $C$ mediated through $B$ is negative (Figure 2, middle panel). The net effect of a compound pathway is computed by multiplying the coefficients along the length of the path. Therefore, if $A$ has a positive effect on $B$, and $B$ has a negative effect on $C$, then the net effect of $A$ on $C$ is negative. Taken all together, the positive direct effect of $A$ on $C$ offsets the negative indirect effect of $A$ on $C$ mediated through $B$. In many cases such as these, the bivariate correlation between $A$ and $C$ is weak and nonsignificant, but this is misleading! The SEM implies that $A$ has multiple effects on $C$. See the R Markdown file for a simulated example.

To make this less abstract, let us turn to an example from the literature. Hall et al. (2009) studied the effect of land use change on nitrogen $(\mathrm{N})$ demand in lake ecosystems. They detected no significant bivariate correlation between land use and $\mathrm{N}$ demand. If the authors would have limited their paper to a simple bivariate correlation, they would have concluded that land use change has no effect on $\mathrm{N}$ demand despite clear theory that would predict land use change to have significant impacts on N cycling. Hall et al. (2009) found from their SEM results that clearing vegetation leads to increased runoff into streams and lakes, which increased $\mathrm{N}$ availability and therefore lowered $\mathrm{N}$ demand. However, changing land use also increased primary production in the lakes, which simultaneously increased $\mathrm{N}$ demand. These two pathways that linked land use change to $\mathrm{N}$ demand were offsetting, creating a bivariate relationship that looks like nothing is going on. This does not mean that there was no effect of land use change on $\mathrm{N}$ demand.
Quite the contrary! The multivariate analysis uncovered a set of indirect effects that illustrate how changing land use has counteracting effects on $\mathrm{N}$ cycling. This exemplifies the importance of offsetting effects in ecological systems.

We believe that the distrust in these results among ecologists is rooted in our long-standing dependence on univariate statistics. If two variables are uncorrelated, our first instinct is to think they have no causal connection. This is actually the inverse problem of overinterpreting correlations to imply a causation. In the case of offsetting effects, a nonsignificant bivariate correlation does not imply a lack of relationship between two variables. There are now numerous examples in the literature where a factor has a positive indirect effect on a response that is offset by a negative indirect effect, leading to no net effect (Alsterberg et al. 2013, Wallace et al. 2018). The identification of these networks is an important part of improving our understanding of complex systems.

\section{Suppressed effects}

The only other effect that ecologists seem to distrust even more than offsetting effects are suppressed effects. Suppressed effects occur when the bivariate correlation is, say, positive, but the estimated coefficient in the SEM is quite different, sometimes even the opposite sign. Scientists are often alarmed when simple bivariate correlations differ from those that are estimated in the context of a multivariate model, but the latter are more likely to represent the truth because confounding factors have been statistically controlled. According to one of the most thorough and classic mathematical descriptions of this phenomenon by Cohen and Cohen (1983), this is called 'suppression' because the relationship between the independent variables is hiding or suppresses their real relationship with the response variables, which would be larger or of opposite sign if the independent variables were not correlated. This is illustrated in Figure 2, bottom panel, where the bivariate correlation of $A$ and $C$ is positive, but the sign of the regression coefficient linking $A$ and $C$ in the SEM is negative.

We were surprised by the discovery of a suppressed effect during an analysis of fire history effects on plant diversity in an old-growth ponderosa pine forest (Laughlin and Grace 2006). Frequently burned forests exhibited shorter time intervals since they last burned. The bivariate correlation between fire frequency and understory species richness was positive, which we deemed plausible given the long evolutionary history of fire in this ecosystem. However, once we statistically controlled for time-since-fire (another important attribute of the disturbance regime), model results implied that areas with more frequent fires had lower richness than otherwise expected. In short, the sign of the relationship 
switched from positive to negative. We interpreted the negative association of frequent fires with understory richness as an indication that multiple fires can actually harm the understory, possibly by depletion of soil $\mathrm{N}$ after repeated exposure to surface fire. See the R Markdown file for a simulated example based on this analysis.

Tanentzap et al. (2014) studied the effects of flooding, soil fertility, and grazing on non-native plant invasion in an ephemeral wetland in New Zealand. They showed that the bivariate correlation between invasion and soil fertility was negative, which they could have simply interpreted as invasion being greatest where soil nutrient availability is lowest. However, in the context of their multivariate SEM, Tanenztap et al. (2014) discovered that fertility had a positive effect on invasion after controlling for the effects of flooding and grazing, which made sense given the high nutrient requirement of the invasive plants.

\section{Additional Possibilities}

It is worth mentioning some additional types of applications that are possible and that demonstrate the flexibility SEM. Its flexibility is a general attribute of SEM that users report as beneficial. Most quantitative methods summarize results in very specific, predetermined form; SEM can be used to integrate such results into an explanatory network of relationships. As an example, ecologists often deal with a great deal of biological complexity and then want to incorporate summaries of those complex relationships into their system-level analyses. Delgado-Baquerizo et al. (2018) examined soil microorganisms from a continent-scale survey including 647 sites across 58 degrees of latitude between the Australian tropics and Antarctica. Using network analysis, they found six major ecological clusters (modules) of co-occurring soil taxa and then incorporated the modules into evaluations of hypotheses. Using this approach, they were able to obtain new insights into the mechanisms driving soil biodiversity.

Another important flexibility of SEM is the ability to integrate observational with experimental data. Conventional analyses of experimental data, such as ANOVA, are only able to estimate a net effect and only provide inferences for the treatment manipulation (e.g., the addition of fertilizer or fencing to exclude herbivores). Inferences about the causative agents of interest are often not supported when using ANOVA, as the causes of interest, like soil nutrient levels or quantities of vegetation removed by herbivores, are neither controlled or randomized. SEM can allow the investigator to integrate the system properties that are of causal inference into a network hypothesis where indirect effects through mediator variables provide demonstrations of mechanisms, as well as discovery of new mechanisms. Using such an approach, Whalen et al. (2013) were able to reveal a network of interactions between covariates and mediators in their study of marine food webs.

On another front, SEM is starting to be used by ecologists to predict ecological attributes into the future. Roland et al. (2019) examined the drivers of high and low diversity microenvironments in Alaska and found a number of regulating factors. Disentangling climate influences from other controls allowed them to suggest where major consequences from warming trends might have the greatest impacts on biodiversity. It is natural to segue from retrospective explanations to implications for future changes and SEM provides a helpful framework for both enterprises.

Finally, we anticipate that future applications of the SEM framework will continue to take advantage of opportunities to incorporate new statistical techniques into SE models. Grace et al. (2012) provide an overview of this ongoing evolution, highlighting nonlinear modeling as a pressing need and Bayesian estimation methods as one of several means of increasing modeling flexibility. At present, there is great interest in multi-level modeling and software development is aiding in this endeavor. Because SEM constitutes a framework, there are few limitations to the potential to combine with specific quantitative techniques.

\section{Conclusions}

Survey results suggest that those with experience using SEM end up having a clear recognition of the multiple ways that standard regression analyses provide incomplete and even misleading inference about systems. The great majority of survey respondents (all but one individual) stated the belief that SEM should be part of the tool box for all ecologists. In our experience, one problem with achieving that goal is getting the word out, as this paper attempts to do. A related problem at the moment is a deficit in training in SEM. Statisticians seem drawn to descriptive methods where issues related to causal inference can be ignored and one can focus on the mathematical issues. This leaves it to scientists to become cross-trained so as to become the experts in SEM. We hope these examples convince ecologists that this effort is valuable and even necessary to advance ecology.

\section{Data Accessibility}

No real data was used in this paper. $R$ code that simulates data to illustrate all of these examples are provided in the following github repository: https://github.com/danielLaughlin/semDiscoveries 


\section{Acknowledgements}

JG was supported by the USGS Land Change Science and Ecosystems Programs. Any use of trade, firm, or product names is for descriptive purposes only and does not imply endorsement by the U.S. Government.

\section{Referees}

Manuel Delgado-Baquerizo

m.delgadobaquerizo@gmail.com

University of Colorado at Boulder

Antonio Gama-Rodrigues - tonygama@uenf.br

Universidade Estadual do Norte Fluminense

\section{References}

Alsterberg, C., Eklöf, J.S., Gamfeldt, L., Havenhand, J.N., and K. Sundbäck. 2013. Consumers mediate the effects of experimental ocean acidification and warming on primary producers. Proceedings of the National Academy of Sciences: 201303797. CrossRef

Chen, D., Lan, Z., Bai, X., Grace, J.B., and Y. Bai. 2013. Evidence that acidification-induced declines in plant diversity and productivity are mediated by changes in below-ground communities and soil properties in a semi-arid steppe. Journal of Ecology 101:1322-1334. CrossRef

Clark, C.M., Cleland, E.E., Collins, S.L., Fargione, J.E., Gough, L., Gross, K.L., et al. 2007. Environmental and plant community determinants of species loss following nitrogen enrichment. Ecology Letters 10:596-607. CrossRef

Cohen, J., and C. Cohen. 1983. Applied multiple regression/correlation analysis for the behavorial sciences. Taylor and Francis Group, New York.

Delgado-Baquerizo, M., Reith, F., Dennis, P.G., Hamonts, K., Powell, J.R., Young, A., et al. 2018. Ecological drivers of soil microbial diversity and soil biological networks in the Southern Hemisphere. Ecology 99:583-596. CrossRef

Fox, J. 2015. Applied regression analysis and generalized linear models. Sage Publications.

Godschalx, A.L., Schädler, M., Trisel, J.A., Balkan, M.A., and D.J. Ballhorn. 2015. Ants are less attracted to the extrafloral nectar of plants with symbiotic, nitrogen-fixing rhizobia. Ecology 96:348-354. CrossRef

Gough, L., and J.B. Grace. 1999. Effects of environmental change on plant species density: comparing predictions with experiments. Ecology 80:882-890. CrossRef
Grace, J.B. 2006. Structural Equation Modeling and Natural Systems. Cambridge University Press. CrossRef

Grace, J.B., Adler, P.B., Stanley Harpole, W., Borer, E.T., and E.W. Seabloom. 2014. Causal networks clarify productivity-richness interrelations, bivariate plots do not. Functional Ecology 28:787-798. CrossRef

Grace, J.B., Schoolmaster, D.R., Guntenspergen, G.R., Little, A.M., Mitchell, B.R., Miller, K.M., et al.. 2012. Guidelines for a graph-theoretic implementation of structural equation modeling. Ecosphere 3:144. CrossRef

Grace, J.B., Youngblood, A., and S.M. Scheiner. 2009. Structural equation modeling and ecological experiments. Pages 19-45 Real World Ecology. Springer. $\underline{\text { CrossRef }}$

Hall, R.O., Tank, J.L., Sobota, D.J., Mulholland, P.J., O'Brien, J.M., Dodds, W.K., et al. 2009. Nitrate removal in stream ecosystems measured by $15 \mathrm{~N}$ addition experiments: Total uptake. Limnology and Oceanography 54:653-665. CrossRef

Laughlin, D.C., and J.B. Grace. 2006. A multivariate model of plant species richness in forested systems: old-growth montane forests with a long history of fire. Oikos 114:60-70. CrossRef

Lortie, C.J. 2018. A comment on computational biology and connecting the dots. Ideas in Ecology and Evolution 10:86-89. CrossRef

Roland, C.A., Sadoti, G., Nicklen, E.F., McAfee, S.A., and S.E. Stehn. 2019. A structural equation model linking past and present plant diversity in Alaska: a framework for evaluating future change. Ecosphere 10:e02832. CrossRef

Shipley, B. 2002. Cause and correlation in biology: a user's guide to path analysis, structural equations and causal inference. Cambridge University Press.

Tanentzap, A.J., Lee, W.G., Monks, A., Ladley, K., Johnson, P.N., Rogers, G.M., et al. 2014. Identifying pathways for managing multiple disturbances to limit plant invasions. Journal of Applied Ecology 51:10151023. CrossRef

Wallace, K.J., Laughlin, D.C., Clarkson, B.D., and L.A. Schipper. 2018. Forest canopy restoration has indirect effects on litter decomposition and no effect on denitrification. Ecosphere 9:e02534. CrossRef

Whalen, M.A., Duffy, J.E., and J.B. Grace. 2013. Temporal shifts in top-down vs. bottom-up control of epiphytic algae in a seagrass ecosystem. Ecology 94:510-520. $\underline{\text { CrossRef }}$ 